\title{
Causes and Effects of Filament Eruptions
}

\author{
Boris P. Filippov \\ Institute of Terrestrial Magnetism, Ionosphere and Radio Wave \\ Propagation, Russian Academy of Sciences, 142092, Troitsk Moscow \\ Region, Russia
}

\begin{abstract}
Observational and theoretical aspects of filament destabilization are reviewed in light of the concept of filament eruption as catastrophic loss of equilibrium in a magnetic configuration of inverse polarity. Interrelations between eruptive prominences and other phenomena of solar activity such as flares, CMEs, and loop arcade are considered.
\end{abstract}

\section{Energetics of Filament Equilibrium}

In spite of the fact that filaments are perhaps the most long-lived and stable features in the solar atmosphere, at times they either demonstrate fast changes and motions, or disappear completely from view. Sometimes the activation of a filament is initiated by appreciable external events, for example by a remote flare or fast emergence of new magnetic flux nearby, but more often motion of a filament begins before other manifestations of energetic non-stationary processes in the atmosphere of the Sun. In the majority of cases, there is no reliable evidence in favor of an external source of filament destabilization.

The cause of eruption is more likely not in a strong external action but in properties of filament equilibrium itself, possibly in the rapid growth of instability. Since there appears to be little doubt that the prominence material is kept from falling by the magnetic field, the causes of eruption should be searched for in peculiarities of the magnetic configuration around the filament. In this connection it should be remembered that there are two possible directions of a filament current relative to the direction of the field of subphotospheric sources. The external field can support a current if the force is directed upwards, or press a current to the photosphere. In the latter case, the current in a filament should be so great that the currents induced by them on the surface of the photosphere and equivalent to a mirror current are able to generate a magnetic field stronger than the field of subphotospheric sources. This is the configuration of models of inverse polarity, while the former configuration corresponds to models of normal polarity (Priest 1990). The current magnitude is connected to the amount of magnetic energy stored in the corona which can be released and become apparent during the eruption. From the point of view of fast eruptions, the inverse and normal configurations are not equivalent. Simple force balance shows that for a filament of normal polarity the Lorenz force is equal to the weight of the filament

$$
\frac{I B}{c}=m g,
$$


and, in the case of the disturbance of equilibrium assuming reasonable conditions, $\Delta B \ll B, \Delta I \ll I$, the acceleration of the filament can reach only a small fraction of the free-fall acceleration, $g$. In inverse polarity models, an equilibrium is possible in which the weight of a filament is of minor importance

$$
\frac{I^{2}}{h c^{2}}=\frac{I B}{c} \gg m g,
$$

where $h$ is the height of the filament above the photosphere. A small violation of equilibrium is able to create an acceleration large compared with $g$.

\section{Changing of External Conditions Before and During an Eruption}

The question arises what changes of magnetic field and photospheric displacements lead to current growth, twist increase, loss of stability and equilibrium of a filament, that is, to eruption. It is obvious that non-uniform vortical motions produce a growing current flow (Brandt et al. 1988, Browning 1991). Simon et al. (1986) provided evidence that the evolution of the small scale photospheric magnetic field near a filament, namely pore movement or a new pore birth, is at the origin of the destabilization of the filament. Feynman and Martin (1995) found from study of 53 filaments observed from September 1991 to February 1992 that there is a high probability that a filament will erupt when major new flux emerges within or adjacent to the unipolar magnetic fields astride a filament in an orientation favorable for reconnection. Flux concellation in the vicinity of a neutral line is assumed by Martin (1986) as a necessary condition for filament formation and eventually for its eruption. Expectations following from theoretical notions may at times make a pattern of observed motions seem more ordered than it actually is. One type of field deformation leading to instability most favorite by theorists is shear motion, or moving of the footpoints of arches parallel to a neutral line in opposite direction on both sides. Another type of motion is a converging motion which transports new magnetic flux tubes towards the neutral line to support the conditions for reconnection. Observations confirm, indeed, the presence of such motions near the neutral line (Martin 1986, Athay et al. 1986, Athay 1990), but it is unlikely that one can say with certainty that just these motions are responsible for the growth of the current and loss of filament equilibrium. It is a very telltale fact that the fine structure of filaments contradicts a pattern which would be expected from simple action of differential rotation on a coronal arcade. Although Priest (1996) and Kuperus (1996) proposed some new models of filament magnetic field generation due to differential rotation and flux concellation, these ideas hardly solve the problem completely.

Observational data on movements and magnetic field changes at the photospheric level and eruptive filament motions suggest that the discrepancy of the characteristic times of these processes is as much as two orders of magnitude. It is obvious that a simple linear relationship cannot exist between processes in these two layers. The mechanism which maintains the prominence equilibrium should offer sufficient nonlinearity for catastrophic process to be in progress under small and slow changes of the external conditions. Models of inverse polarity again meet this requirement to a greater extent. In the simplest twodimensional model with a line current, as was noted by Van Tend and Kuperus 
(1978), if the photospheric background field falls off with height faster than $1 / h$, a critical value for a filament current exists. If the current is less than the critical value, a filament is in stable equilibrium. The equilibrium height increases with the growth of a current. When a current exceeds a critical value the equilibrium will be lost. Therefore, smooth evolution of a filament terminates and eruption follows. The characteristics of filament motion will no longer be determined by changes of the photospheric field and will depend only on the spatial distribution of magnetic field in the corona, the filament weight and properties of a circuit. In terms of potential energy, the process looks like gradual reduction of the depth of a potential well with the growth of a current, and its complete flattening when the current achieves the critical value.

A consideration of the third dimension adds helical and kink instabilities inherent in a straight current (Sakurai 1976). Measurements of magnetic fields in prominences (Rust 1967, Leroy et al. 1984) testify that the field vector makes a small angle with the axis of a filament. Therefore, a filament can be represented by a twisted flux tube extended along a neutral line. These flux rope or flux tube models correlate well with the observed internal fine structure of filaments. The helical structure of filaments most clearly manifests itself during eruptions. There are a lot of studies which have investigated the stability of cylindrical helical structures in various environments and at various deformations (Raadu 1972, Hood and Priest 1979, Kuperus and Van Tend 1981, Vrsnak 1990). The theory imposes limits on the maximum value of twist for a filament to be stable. These threshholds are confirmed roughly by the observed twists in quiescent and eruptive filaments (Vrsnak et al. 1991).

\section{Eruptions and $\mathrm{H} \alpha$ Flares}

As a system in equilibrium, a filament should be sensitive to any changes of magnetic field. A filament can be activated by a flare at some distance away from it. The activation can be limited to several oscillations of a filament near the equilibrium position, if it is stable enough (that is its potential well is deep), or it can result in eruption of the filament. More intimate connections occur between eruptions and bright strands arising on both sides of the ascending filament. The connection is so close that both phenomena are, probably, different manifestations of a single process of the reconstruction of the magnetic configuration. Filament eruption begins before the appearance of bright ribbons so, in some sense, it can be considered as "primary".

Many people have outlined a scenario of the process using two-dimensional models of inverse polarity (Kaastra 1985, Martens and Kuin 1989, Priest and Forbes 1990, Forbes and Isenberg 1991, Forbes 1991). Sturrock (1989) has tried to conceive of a three-dimensional geometry of such a process. And his scheme finds some support in numerical calculations of the evolution of a stretchable current contour in external magnetic field (Filippov 1996a). It is possible that ascending filament current creates two factors which provide favorable conditions for energy release in the corona. First, an additional source of magnetic field, represented by filament current located high in the corona, creates readily a saddle structure which is the basic prerequisite for reconnection or builds up a helmet configuration with a current sheet when moving through the corona 


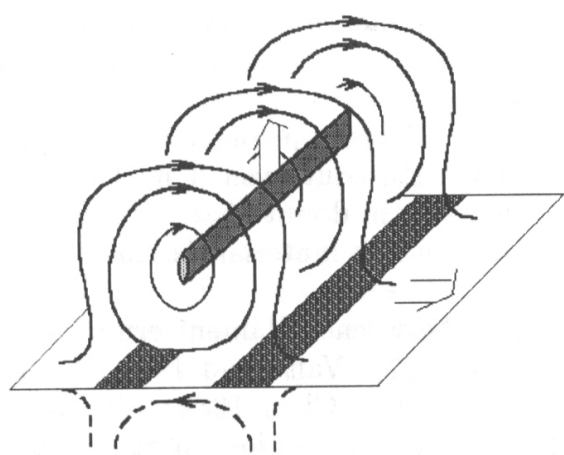

a

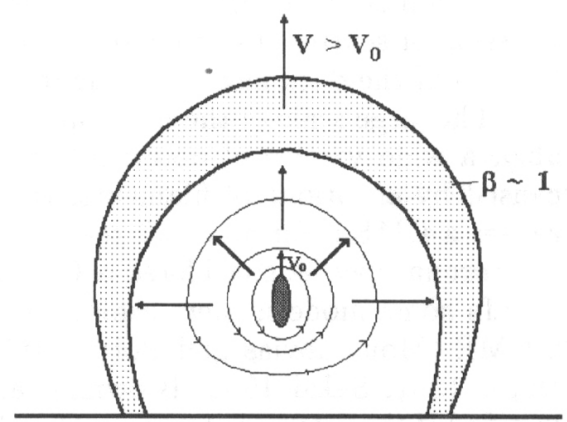

b

Figure 1. Schematic representations of the magnetic configuration with flare ribbons therein (a) and of CME formation (b).

(Kopp and Pneuman 1976, Pneuman 1982). Second, fast movement of such a source produces fast changes in the surrounding magnetic field, which cannot be expected as a result of the relatively slow emergence of new magnetic flux in the photosphere.

In general, a broad spectrum of different effects would be expected during the fast changes of magnetic field with complex topology ranging from acceleration of particles to plasma heating. For example, emission in the ribbons (particularly outside of active regions) can arise as a result of heating of the chromospheric gas compressed by changing magnetic field in the regions where field lines diverge drastically (Filippov 1997). Such regions exist in inverse polarity models on both sides of the filament (Figure 1a).

\section{Eruptive Filaments as a Part of Coronal Mass Ejections}

There is no doubt that an ascending filament current as a source of magnetic field should make an appreciable rearrangement of coronal density in large volume surrounding an eruptive filament. Investigations of coronal mass ejections (CMEs) have revealed eruptive prominences to be the best-correlated phenomena among the other manifestations of activity in low layers of the solar atmosphere (Munro et al. 1979, Webb and Hundhausen 1987, St. Cyr and Webb 1991). In many images obtained by orbital coronagraphs, an eruptive prominence is clearly visible as a bright core of a CME surrounded by a dark cavity of depleted material. The cavity is bordered by a bright loop of enhanced density (Sime et al. 1984, Hundhausen 1998). In these events, it is impossible to deny an interrelation between CMEs and eruptive prominences, but several empirical arguments have been used against the concept that the prominence eruption drives the CME (Hundhausen 1998): (i) There are CMEs which have no bright cores and there are no data on simultaneous filament eruptions or the disappearance of a dark filament from the visible solar disk. (ii) The average angular width of CMEs is about $50^{\circ}$. This scale size is larger than that of erup- 
tive prominences $\sim 20^{\circ}$. (iii) The top of the prominence observed in either $\mathrm{H} \alpha$ emission or scattered white light almost always moves outward more slowly than the top of the dark cavity or bright frontal loop.

The large scale of the phenomenon and the effects of projection favor, probably, a wide variety of observable forms of CMEs. But even if no CMEs are caused by eruptions of filaments, there can be little doubt that each eruption causes a CME if for no other reason than prominence material moving through the corona may be considered a CME.

In some models, there are no distinctions between a filament eruption and a CME (Mouschovias and Poland 1978, Anzer 1978, Van Tend 1979, Yeh and Dryer 1981, Sakai 1982, Browning and Priest 1984, Chen 1989). It may be justified if the internal structure of a CME is not accounted for. In other models (Pneuman 1980, Anzer and Pneuman 1982, Steele and Priest 1989) the motion of the outside part of a CME is considered separately and the driving force is the magnetic pressure created by the eruptive prominence. Three main parts of the internal CME structure are assumed to exist prior to the beginning of an eruption and, after loss of equilibrium, all parts interact with each other. After a rather short stage of acceleration, both the prominence and the loop move with a constant speed, with the speed of the loop being higher than the speed of prominence in accordance with observations.

Smith et al. (1992) investigated density variations of the coronal plasma when a filament is rising. They used vacuum field calculations based on the low value of $\beta$ in the coronal plasma. They came to the conclusion that the magnetic configuration of a model of inverse polarity is not able to cause the observed plasma distribution in the CME. The problem is that the distance between neighboring field lines above the filament at the location of the CME loop is increasing as the current is rising. Hence, rarefaction of coronal matter should occur there instead of compression. Our approach (Filippov and Shilova 1995, Filippov 1996b) differs by taking into account the limited size of the low $\beta$ region. Then, in the region where magnetic pressure is greater than gas pressure, plasma motion can be assumed as a drift motion. Its characteristic is such that the further one gets from the filament, the larger is the drift velocity of the plasma. This type of motion leads to a rarefaction of plasma and formation of a cavity around the filament in accordance with the results of Smith et al. (1992). Near the boundary $\beta=1$, gas pressure begins to decelerate the plasma motion and, as a result, the coronal density increases. Plasma compression near the surface $\beta=1$ leads to formation of a dense envelope which can be identified with the outer loop of a CME (Figure 1b).

Coming back to the objections of Hundhausen (1998), it seems that they can be rejected within the framework of an understanding of eruption as the loss of equilibrium of the magnetic configuration with coronal current flowing along a neutral line. The cold substance of a prominence only outlines this configuration, but it is not of crucial importance in the equilibrium conditions. Therefore, it is possible to imagine the eruption of an almost "empty" flux tube. 


\section{Post-Flare Loop Systems}

In the wake of an eruption and a flare, systems of expanding loops arise which are visible both in $\mathrm{H} \alpha$ and soft X-rays. A few minutes after the initiation of a large flare, dense plasma loops appear in the corona above the neutral line which can be visible in emission or absorption for hours (Bruzek 1964, Rompolt 1993, Schmieder et al. 1994). The loops connect flare ribbons aligned with the neutral line along both sides of it. The system expands initially with a speed up to $50 \mathrm{~km} / \mathrm{sec}$ which is gradually reduced to $1-2 \mathrm{~km} / \mathrm{sec}$. Observations in coronal lines (McCabe, 1973), in UV (Hiei and Widing 1979), in soft X-rays (Švestka 1987) and in radio wavelengths (Velusamy and Kundu 1981) have revealed the existence of hot coronal arcades over the $\mathrm{H} \alpha$ loops.

The relationship between the arcades and eruptive prominences was not originally noted, possibly because many sets of observations did not cover the initial phase of the phenomenon. Later, observational evidence showed that the system was growing in the space previously occupied by disappeared or erupted filament (Martin 1979). They are referred to as 'post-flare loop systems', though sometimes they are observed in the absence of flares and most of them are associated with sudden filament disappearances or eruptions (Rust and Webb 1977). An illustrative example is of a sequence of events on July 30-31, 1992 in simultaneous observations in radio $(17 \mathrm{GHz}$ ), soft X-rays and $\mathrm{H} \alpha$ (Hanaoka et al. 1994).

Theoretical models of the phenomenon are based on the idea of continuous reconnection of open or greatly elongated field lines at the magnetic X-line which moves upward (Carmichael 1964, Sturrock 1966, Hirayama 1974, Kopp and Pneuman 1976, Forbes et al. 1989). The elongation and opening of field lines is due to the filament eruption. The released energy heats up coronal plasma and loops with temperatures approaching $3 \cdot 10^{7} \mathrm{~K}$. Heat conducted along field lines mapping from the reconnection region to the chromosphere ablates chromospheric plasma and creates associated flare ribbons. In loops leaving a zone of reconnection, plasma cools down to chromospheric temperatures and becomes visible in $\mathrm{H} \alpha$. The plasma in the cool flare loops flows down the legs of the loops. As field lines are reconnected, the loops grow in size, and ribbons move away from one another.

\section{Conclusions}

Electric current above the polarity inversion line in the corona, visualized by filament matter, is a very energetic and mobile feature in the solar atmosphere. Filament equilibrium is realized in a nonlinear manner. So, a catastrophic process is possible under slow and smooth changes of external conditions. Filament eruption implies more or less large scale reconstruction of the magnetic configuration in the corona. It can create the field topology necessary for the action of energy release mechanisms and development of such phenomena as flare ribbons and post-flare loop systems. Rapid motion of filament current in the corona produces fast changes in the surrounding magnetic field. These changes are able to cause compression or rarefaction of plasma resulting in brightenings or CME formation. 
Acknowledgments. This work was supported in part by the Russian Foundation for Basic Research (grant 96-02-16285) and Russian State Astronomical Program.

\section{References}

Anzer, U. 1978, Solar Phys., 57, 111

Anzer, U., Pneuman, G.W., 1982, Solar Phys., 79, 129

Athay, R.G. 1990, Solar Phys., 126, 135

Athay, R.G., Jones, H.P., and Zirin, H. 1986, ApJ, 303, 877

Brandt, P.N., Scharmer, G.B., Ferguson, S., Shine, R.A., Tarbell, T.D., and Title, A.M. 1988, Nature, 335, 238

Browning, P.K. 1991, Plasma Phys. and Contr. Fusion 33, 571

Browning, P.K. and Priest, E.R. 1984, Solar Phys., 92, 173

Bruzek, A. 1964, ApJ, 140, 746

Carmichael, H. 1964, in AAS-NASA Symposium on Physics of Solar Flares, (ed.) W.N. Hess, NASA SP-50, p. 451

Chen, J. 1989, ApJ, 338, 453

Feynman, J. and Martin, S.F. 1995, J. Geophys. Res., 100, 3355

Filippov, B.P. 1996a, Astron. Reports, 40, 754

Filippov, B.P. 1996b, A\&A, 313, 277

Filippov, B.P. 1997, A\&A, 324, 324

Filippov, B.P. and Shilova, N.S. 1995, AZh, 72, 222

Forbes, T.G. 1991, Geophys. Astrophys. Fluid Dynamics, 62, 15

Forbes, T.G., Malherbe, J.M., and Priest, E.R. 1989, Solar Phys., 120, 285

Forbes, T.G. and Isenberg, P.A. 1991, ApJ, 373, 294

Hanaoka, Y. et al. 1994, Publ. Astron. Soc. Japan, 46, 205

Hiei, E. and Widing, K.G. 1979, Solar Phys., 61, 407

Hirayama, T. 1974, Solar Phys., 34, 323

Hood, A.W. and Priest, E.R. 1979, Solar Phys., 64, 303

Hundhausen, A.J. 1998, in The Many Faces of the Sun; Scientific Highlights of the Solar Maximum Mission, (eds.) K.T. Strong, J.L.R. Saba, and B.M. Haisch, Springer-Verlag, New York, in press

Kaastra, J. 1985, Solar Flares: an Electrodynamical Model, Thesis, University of Utrecht

Kopp, R.A. and Pneuman, G.W. 1976, Solar Phys., 50, 85

Kuperus, M. and Van Tend, W. 1981, Solar Phys., 71, 125

Kuperus, M. 1996, Solar Phys., 169, 349

Leroy, J.L., Bommer, V., and Sahal-Brechot, S. 1984, A\&A, 131, 33

Martens, P.C.H. and Kuin, N.P.H., 1989, Solar Phys., 122, 263

Martin, S.F. 1979, Solar Phys., 64, 165

Martin, S.F. 1986, in Coronal and Prominence Plasmas, (ed.) A.I. Poland, 
NASA CP-2442, p. 73

McCabe, M.K. 1973, Solar Phys., 30, 439

Mouschovias, T.Ch. and Poland, A.I. 1978, ApJ, 220, 675

Munro, R.H., Gosling, J.T., Hildner, E., Mc Queen, R.M., Poland, A.E., and Ross, C.L. 1979, Solar Phys., 61, 201

Pneuman, G.W. 1980, Solar Phys., 65, 369

Pneuman, G.W. 1982, Solar Phys., 78, 299

Priest, E.R. 1990, in Dynamics of Quiescent Prominences, (eds.) V. Ruzdjak and E. Tandberg-Hanssen, Springer-Verlag, New York, p. 150

Priest, E.R. 1996, in Solar Drivers of Interplanetary and Terrestrial Disturbances, (eds.) K.S. Balasubramaniam, S.L. Keil, and N. Smartt, ASP Conf. Ser., Vol. 95, p. 229

Priest, E.R. and Forbes, T.G. 1990, Solar Phys., 126, 319

Raadu, M.A. 1972, Solar Phys., 22, 425

Rompolt, B. 1993, Solar Phys., 141, 1

Rust, D.M. 1967, ApJ, 150, 313

Rust, D.M. and Webb, D.F. 1977, Solar Phys., 54, 403

Sakai, J. 1982, ApJ, 263, 970

Sakurai, T. 1976, Publ. Astron. Soc. Japan, 28, 177

Sime, D.G., MacQueen, R.M., and Hundhausen, A.J. 1984, J. Geophys. Res., 89,2113

Simon, G., Gesztelyi, L., Schmieder, B., and Main, N. 1986, in Coronal and Prominence Plasmas, (ed.) A.I. Poland, NASA CP-2442, p. 229

Schmieder, B. et al. 1994, Solar Phys., 150, 199

Smith, D.F., Hildner, E., and Kuin, N.P.M. 1992, Solar Phys., 137, 317

Sturrock, P.A. 1966, Nature, 211, 695

St. Cyr, O.C. and Webb, D.F. 1991, Solar Phys., 136, 379

Steele, C.D.C. and Priest, E.R. 1989, Solar Phys., 119, 157

Sturrock, P. 1989, Solar Phys., 121, 387

Švestka, Z. 1987, Solar Phys., 108, 411

Van Tend, M. 1979, Solar Phys., 61, 89

Van Tend, M. and Kuperus, W. 1978, Solar Phys., 59, 115

Velusamy, T. and Kundu, M.R. 1981, ApJ, 240, 1130

Vrsnak, B. 1990, Solar Phys., 129, 295

Vrsnak, B., Ruzdjak, V., and Rompolt, B. 1991, Solar Phys., 136, 151

Webb, D.F. and Hundhausen, A.J. 1987, Solar Phys., 108, 383

Yeh, T. and Dryer, M. 1981, Solar Phys., 71, 141 